\title{
Palladium(II)-Catalyzed Three-Component Coupling Reaction Initiated by Acetoxypalladation of Alkynes: An Efficient Route to $\gamma, \delta$-Unsaturated Carbonyls
}

\author{
Ligang Zhao and Xiyan Lu*
}

State Key Laboratory of Organometallic Chemistry, Shanghai Institute of Organic Chemistry,

Chinese Academy of Sciences, 354 Fenglin Lu, Shanghai, 200032, China

xylu@pub.sioc.ac.cn

\section{Supporting Information}

Typical Procedure for the Coupling Reaction of 1a and Acrolein (Table 1, entry 1): To a solution of $\mathrm{Pd}(\mathrm{OAc})_{2}(5.6 \mathrm{mg}, 0.025 \mathrm{mmol})$ and 2,2'bipyridine (4.7 $\mathrm{mg}, 0.030 \mathrm{mmol})$ in $\mathrm{HOAc}(1 \mathrm{~mL})$ and $\mathrm{MeCN}(3 \mathrm{~mL})$ at $80{ }^{\circ} \mathrm{C}$ was added $\mathbf{1 a}(98 \mathrm{mg}$, $1 \mathrm{mmol})$ and acrolein $(0.67 \mathrm{~mL}, 10 \mathrm{mmol})$ with stirring. The reaction mixture was monitored by TLC. After the reaction was complete, ethyl ether $(20 \mathrm{~mL})$ was added. On cooling, the reaction mixture was neutralized with saturated $\mathrm{NaHCO}_{3}$, then extracted with $\mathrm{Et}_{2} \mathrm{O}$. The combined ether solution was washed with saturated $\mathrm{NaCl}$, dried $\left(\mathrm{Na}_{2} \mathrm{SO}_{4}\right)$ and concentrated. The residue was purified by flash chromatography $($ EtOAc: petroleum ether $=1: 4)$ to give the coupling products in $72 \%$ yield $(\mathbf{4 a}: \mathbf{5 a}>$ $97: 3)$<smiles>CC(=O)CCC(Cc1ccccc1)=C(C)Cl</smiles>

(Z)-6-Chloro-5-benzyloxycarbonylhept-5-en-2-one 
Oil. ${ }^{1} \mathrm{H}$ NMR $\left(300 \mathrm{MHz}, \mathrm{CDCl}_{3}\right): \delta$ 7.47-7.30 (m, 5H), $5.23(\mathrm{~s}, 2 \mathrm{H}), 2.66-2.48(\mathrm{~m}$, 4H), 2.21 (s, 3H), 2.08 (s, 3H); IR (neat): $v$ 2920, 1730, 1420, 1370, 1285, 1160, 1080, 1015, 750, $700 \mathrm{~cm}^{-1}$; MS (EI): $m / z: 283\left[\mathrm{M}^{+}\left({ }^{37} \mathrm{Cl}\right)+1\right], 281\left[\mathrm{M}^{+}\left({ }^{35} \mathrm{Cl}\right)+1\right], 245,222$, 173, 129, 104, 91 (100), 77, 65, 43; HRMS Calcd for $\mathrm{C}_{15} \mathrm{H}_{17} \mathrm{ClO}_{3}\left[\mathrm{M}^{+}\left({ }^{35} \mathrm{Cl}\right)\right]$ 280.0866 , found 280.0908 .

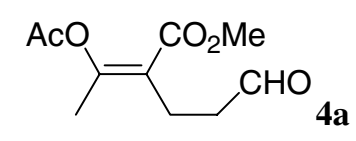

\section{(Z)-5-Acetoxy-4-methoxycarbonylhex-4-enal}

Oil. ${ }^{1} \mathrm{H}$ NMR (300 MHz, $\left.\mathrm{CDCl}_{3}\right): \delta 9.73$ (s, 1H), 3.65 (s, 3H), 2.59-2.56 (m, 4H), 2.17 (s, 3H), $2.05(\mathrm{~s}, 3 \mathrm{H}) ;{ }^{13} \mathrm{C} \mathrm{NMR}\left(75 \mathrm{MHz}, \mathrm{CDCl}_{3}\right): \delta 201.0,168.7,165.9,154.4,118.8$ 51.82, 43.0, 21.5, 20.9, 18.6; IR (neat): $v$ 2955, 2732, 1762, 1723, 1655, 1372, 1231 , 1172, 1090, $1016 \mathrm{~cm}^{-1}$; MS (EI): m/z: (M+1), 215, 173, 155, $154\left(\mathrm{M}^{+}-\mathrm{HOAc}\right), 129$, 116, 97, 43 (100). HRMS Calcd for $\mathrm{C}_{8} \mathrm{H}_{10} \mathrm{O}_{3}\left[\mathrm{M}^{+}\right.$-HOAc] 154.0630, found 154.0616.

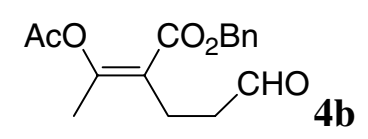

\section{(Z)-5-Acetoxy-4-benzyloxycarbonylhex-4-enal}

Oil. ${ }^{1} \mathrm{H}$ NMR (300 MHz, $\mathrm{CDCl}_{3}$ ): $\delta 9.77$ (s, 1H), 7.38-7.27 (m, 5H), 5.15 (s, 2H), 2.65 (s, 4H), $2.04(\mathrm{~s}, 3 \mathrm{H}), 1.88(\mathrm{~s}, 3 \mathrm{H}) ;{ }^{13} \mathrm{C} \mathrm{NMR}\left(75 \mathrm{MHz}, \mathrm{CDCl}_{3}\right): \delta 200.9,168.6,165.4$ $154.4,135.5,128.6,128.4,128.3,118.9,66.7,42.9,21.5,20.5,18.6$; IR (neat): v 3036, 2956, 2729, 1760, 1721, 1654, 1499, 1456, 1226, 1168, 1015, 1081, 755, $700 \mathrm{~cm}^{-1}$ MS (EI): $m / z: 291\left(\mathrm{M}^{+}+1\right), 230\left(\mathrm{M}^{+}-\mathrm{HOAc}\right), 139,141,92,91(100), 77,65,43 ; \mathrm{HRMS}$ 
Calcd for $\mathrm{C}_{14} \mathrm{H}_{14} \mathrm{O}_{3}\left[\mathrm{M}^{+}\right.$-HOAc] 230.0943, found 230.0905.

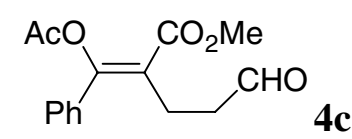

(Z)-5-Acetoxy-4-methoxycarbonyl-5-phenylpent-4-enal

Oil. ${ }^{1} \mathrm{H}$ NMR (300 MHz, $\left.\mathrm{CDCl}_{3}\right): \delta 9.68(\mathrm{~s}, 1 \mathrm{H}), 7.41-7.39(\mathrm{~m}, 5 \mathrm{H}), 3.79(\mathrm{~s}, 3 \mathrm{H})$, 2.67-2.62 (m, 4H), $2.16(\mathrm{~s}, 3 \mathrm{H})$; IR (neat): v 2954, 2841, 2730, 1765, 1722, 1648 , 1492, 1436, 1370, 1326, 1207, 890, $701 \mathrm{~cm}^{-1}$; MS (EI): $m / z: 277\left(\mathrm{M}^{+}+1\right), 233$, 217(100), $216\left(\mathrm{M}^{+}\right.$-HOAc), 105, 43; HRMS Calcd for $\mathrm{C}_{14} \mathrm{H}_{12} \mathrm{O}_{3}\left[\mathrm{M}^{+}\right.$-HOAc] 216.0786, found 216.0794.

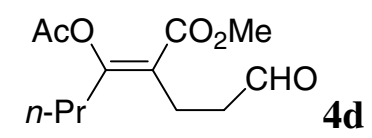

\section{(Z)-5-Acetoxy-4-methoxycarbonyloct-4-enal}

Oil. ${ }^{1} \mathrm{H}$ NMR (300 MHz, $\left.\mathrm{CDCl}_{3}\right): \delta 9.80(\mathrm{~s}, 1 \mathrm{H}), 3.71(\mathrm{~s}, 3 \mathrm{H}), 2.65(\mathrm{~s}, 4 \mathrm{H}), 2.34(\mathrm{t}, J=$ $7.56 \mathrm{~Hz}, 2 \mathrm{H}), 2.05(\mathrm{~s}, 3 \mathrm{H}), 1.54(\mathrm{dt}, J=7.56 \mathrm{~Hz}, J=7.49 \mathrm{~Hz}, 2 \mathrm{H}), 0.96$ ( t, $J=7.49$ Hz, 3H); IR (neat): v 2966, 2729, 1763, 1723, 1647, 1436, 1370, 1236, 1222, 1168 , 1099, 1060, $929 \mathrm{~cm}^{-1}$; MS (EI): m/z: $182\left(\mathrm{M}^{+}\right.$-HOAc), 169, 157, 144, 125, 116, 71, 43(100); HRMS Calcd for $\mathrm{C}_{10} \mathrm{H}_{14} \mathrm{O}_{3}$ [ $\mathrm{M}^{+}$-HOAc] 182.0943, found, 182. 0920.

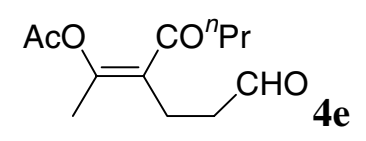

(Z)-5-Acetoxy-4-butyrylhex-4-enal

Oil. ${ }^{1} \mathrm{H}$ NMR (300 MHz, $\left.\mathrm{CDCl}_{3}\right): \delta 9.77(\mathrm{~s}, 1 \mathrm{H}), 2.60-2.51(\mathrm{~m}, 4 \mathrm{H}), 2.19(\mathrm{~s}, 3 \mathrm{H}), 2.05$ 
(s, 3H), 1.64-1.59 (m, 4H), 0.97-0.89 (m, 3H); IR (neat): v 2966, 2877, 2729, 1761, 1724, 1665, 1459, 1372, 1018, $898 \mathrm{~cm}^{-1}$; MS (EI): m/z: $227\left(\mathrm{M}^{+}+1\right), 166\left(\mathrm{M}^{+}-\mathrm{HOAc}\right)$, 141, 128, 100, 71, 43(100); HRMS Calcd for $\mathrm{C}_{10} \mathrm{H}_{14} \mathrm{O}_{2}\left[\mathrm{M}^{+}\right.$-HOAc] 166.0904, found 166.0985 .

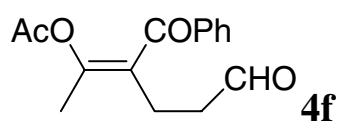

\section{(Z)-5-Acetoxy-4-benzoylhex-4-enal}

Oil. ${ }^{1} \mathrm{H}$ NMR (300 MHz, $\left.\mathrm{CDCl}_{3}\right): \delta 9.79(\mathrm{~s}, 1 \mathrm{H}), 7.79-7.76(\mathrm{~m}, 2 \mathrm{H}), 7.53-7.52(\mathrm{~m}$, 1H), 7.47-7.40 (m, 2H), 2.70-2.68 (m, 4H), $2.16(\mathrm{~s}, 3 \mathrm{H}), 1.51(\mathrm{~s}, 3 \mathrm{H})$; IR (neat): $v$ 3063, 2831, 2731, 1757, 1724, 1660, 1598, 1580, $1449 \mathrm{~cm}^{-1}$; MS (EI): m/z: $200\left(\mathrm{M}^{+}-\right.$ HOAc), 199, 105, 162, 97, 43(100); HRMS Calcd for $\mathrm{C}_{13} \mathrm{H}_{12} \mathrm{O}_{2}\left[\mathrm{M}^{+}\right.$-HOAc] 200.0837, found 200.0804 .

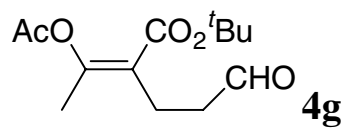

\section{(Z)-5-Acetoxy-4-tert-butoxycarbonylhex-4-enal}

Oil. ${ }^{1} \mathrm{H}$ NMR (300 MHz, $\mathrm{CDCl}_{3}$ ): $\delta 9.80$ (s, 1H), 2.66-2.56 (m, 4H), 2.16 (s, 3H), 1.99 (s, 3H), 1.46 (s, 9H); IR (neat): v 2980, 2729, 1762, 1717, 1658, 1370, 1233, 1153, 1087, $849 \mathrm{~cm}^{-1}$; MS (EI): m/z: $257\left(\mathrm{M}^{+}+1\right), 201(100), 183\left(\mathrm{M}^{+}-\mathrm{O}^{t} \mathrm{Bu}\right), 141,43,41$; HRMS Calcd for $\mathrm{C}_{9} \mathrm{H}_{11} \mathrm{O}_{4}\left[\mathrm{M}^{+}-\mathrm{O}^{t} \mathrm{Bu}\right]$ 183.0657, found 183.0661. 


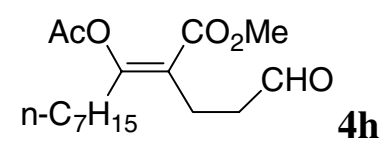

\section{(Z)-5-Acetoxy-4-methoxycarbonyldodec-4-enal}

Oil. ${ }^{1} \mathrm{H}$ NMR (300 MHz, $\left.\mathrm{CDCl}_{3}\right): \delta 9.80(\mathrm{~s}, 1 \mathrm{H}), 3.71(\mathrm{~s}, 3 \mathrm{H}), 2.65(\mathrm{~s}, 4 \mathrm{H}), 2.35(\mathrm{t}, J=$ 7.4Hz 2H), $2.19(\mathrm{~s}, 3 \mathrm{H}), 1.49-1.46(\mathrm{~m}, 2 \mathrm{H}), 1.33-1.28(\mathrm{~m}, 8 \mathrm{H}), 0.88(\mathrm{t}, J=6.4 \mathrm{~Hz}$ 3H); IR (neat): v 2965, 2858, 2726, 1764, 1723, 1646, 1460, 1436, 1369, 1227, 1167 , 1102, $923 \mathrm{~cm}^{-1}$; MS (EI): m/z: 266, 238 (M+HOAc), 254(100), 225, 213, 41; HRMS Calcd for $\mathrm{C}_{14} \mathrm{H}_{22} \mathrm{O}_{3}\left[\mathrm{M}^{+}\right.$-HOAc] 238.1569, found 238.1591.

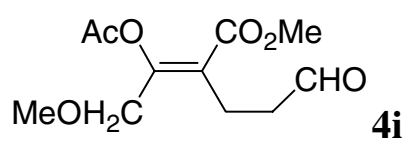

\section{(Z)-5-Acetoxy-4-methoxycarbonyl-6-methoxyhex-4-enal}

Oil. ${ }^{1} \mathrm{H}$ NMR (300 MHz, $\left.\mathrm{CDCl}_{3}\right): \delta 9.91(\mathrm{~s}, 1 \mathrm{H}), 4.28(\mathrm{~s}, 2 \mathrm{H}), 3.87$ (s, 4H), 3.48 (s, 3H), 2.81 (s, 3H), 2.00 (s, 3H); IR (neat): v 2954, 2829, 2733, 1764, 1724, 1655, 1437 , 1371, 1319, 1226, 1171, 1084, $922 \mathrm{~cm}^{-1}$; MS (EI): m/z: $245\left(\mathrm{M}^{+}+1\right), 185\left(\mathrm{M}^{+}-\mathrm{OAc}\right)$ 171, 152, 142, 125, 127, 43(100); HRMS Calcd for $\mathrm{C}_{9} \mathrm{H}_{13} \mathrm{O}_{4}\left[\mathrm{M}^{+}-\mathrm{OAc}\right]$ 185.0814, found 185.0768 .

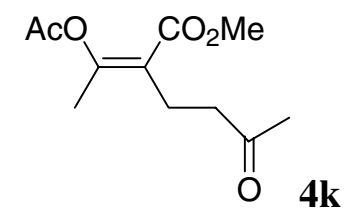

\section{(Z)-6-Acetoxy-5-methoxycarbonylhept-5-en-2-one}

Oil. ${ }^{1} \mathrm{H}$ NMR (300 MHz, $\left.\mathrm{CDCl}_{3}\right): \delta 3.72(\mathrm{~s}, 3 \mathrm{H}), 2.65-2.55(\mathrm{~m}, 4 \mathrm{H}), 2.17$ (s, 3H), 2.16 (s, 3H), 2.04 (s, 3H); IR (neat): v 2955, 1762, 1720, 1656, 1437, 1369, 1230, 1167 
$\mathrm{cm}^{-1}$; MS (EI): m/z: 197, 187, $168\left(\mathrm{M}^{+}-\mathrm{HOAc}\right), 155,129,97,43(100)$; HRMS Calcd for $\mathrm{C}_{9} \mathrm{H}_{12} \mathrm{O}_{3}\left[\mathrm{M}^{+}-\mathrm{HOAc}\right]$ 168.0786, found 168.0780. 


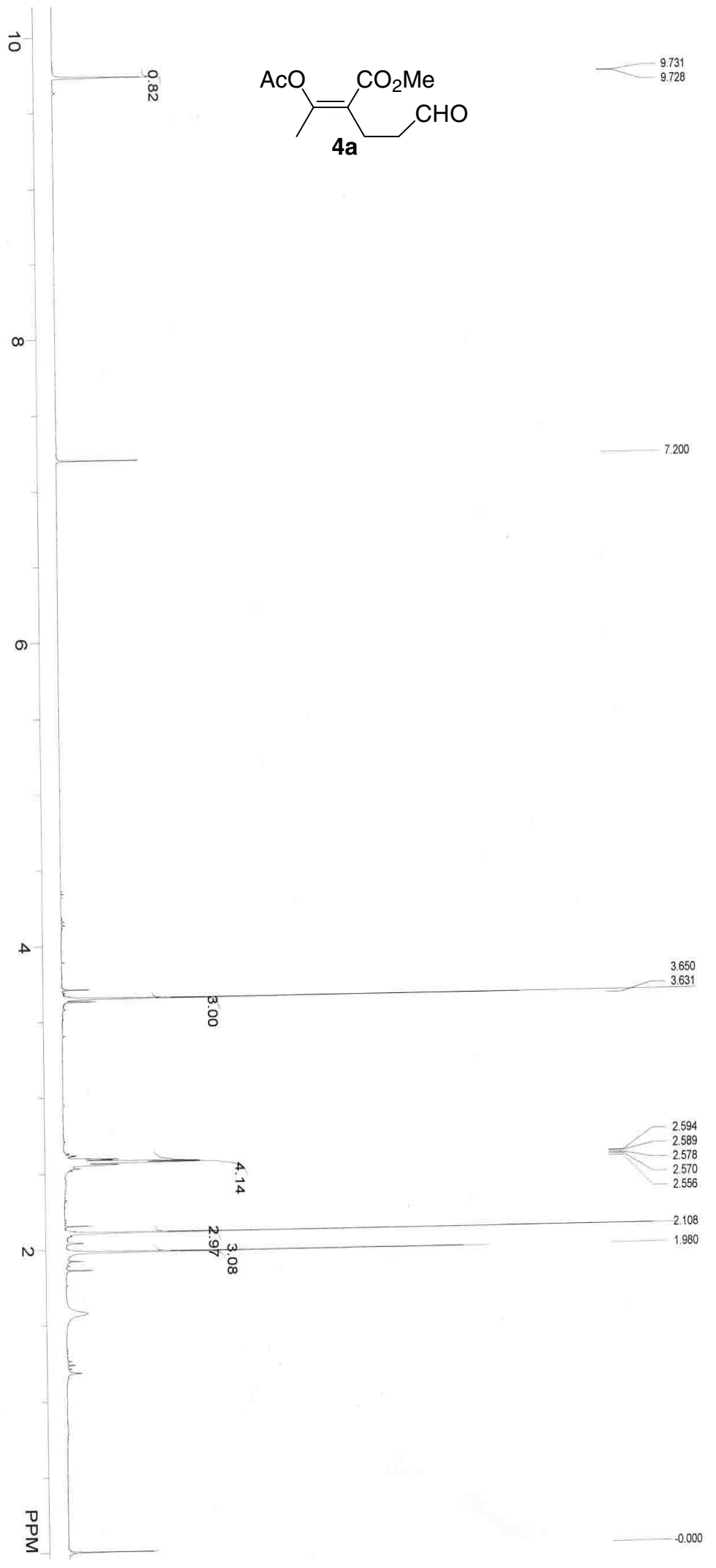



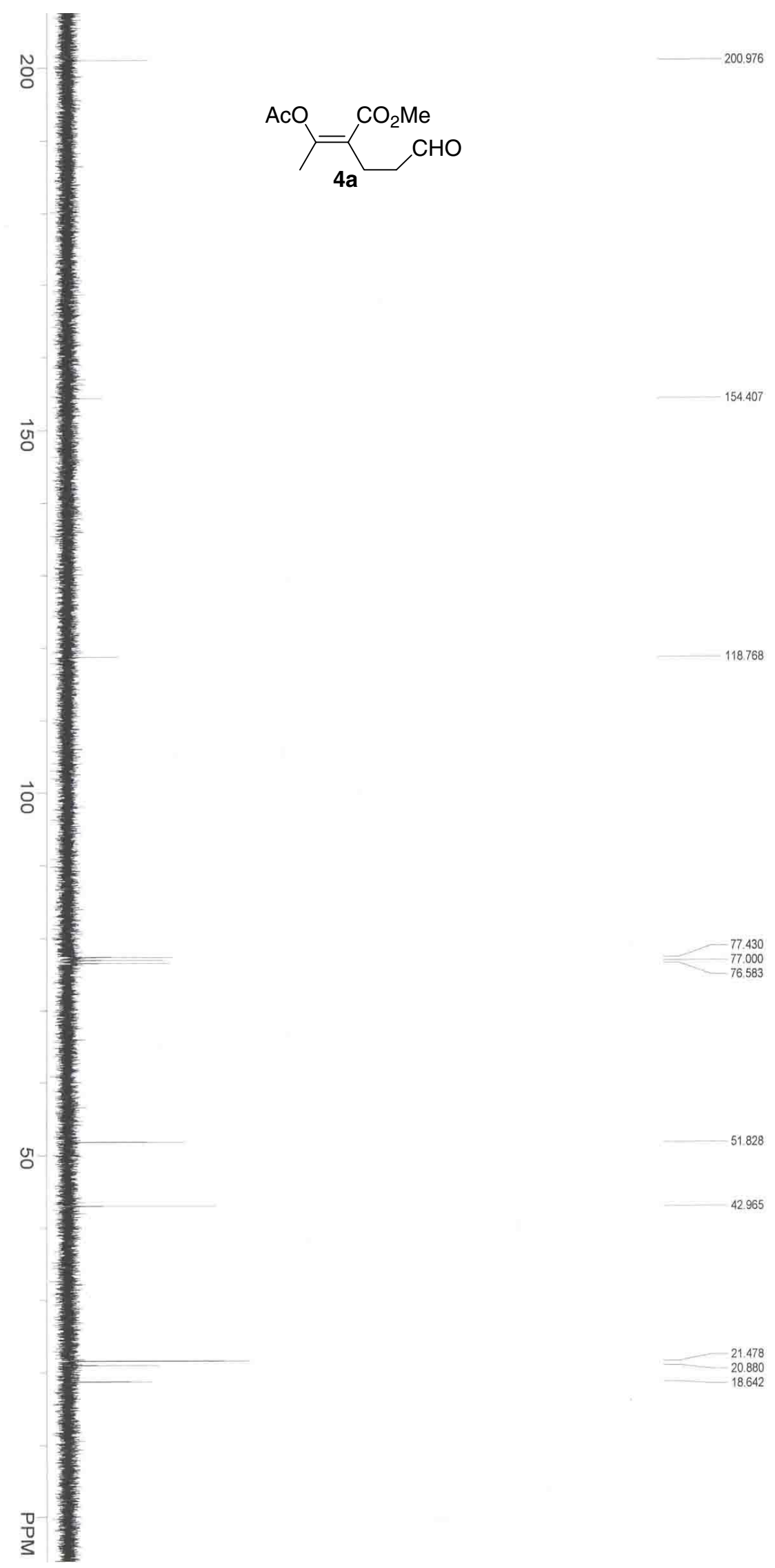

$-51.828$ 42.965

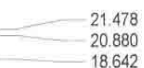




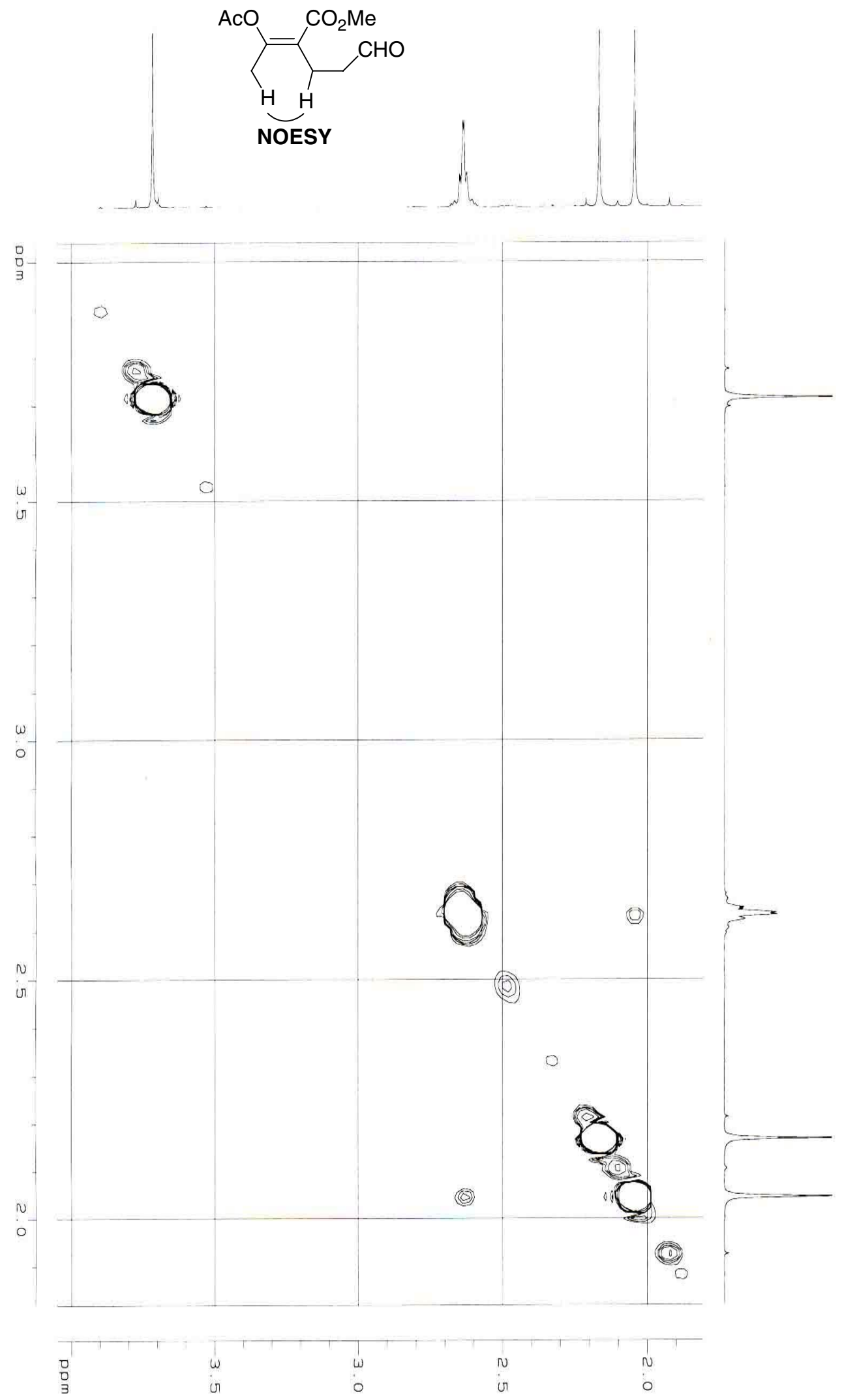

S9 

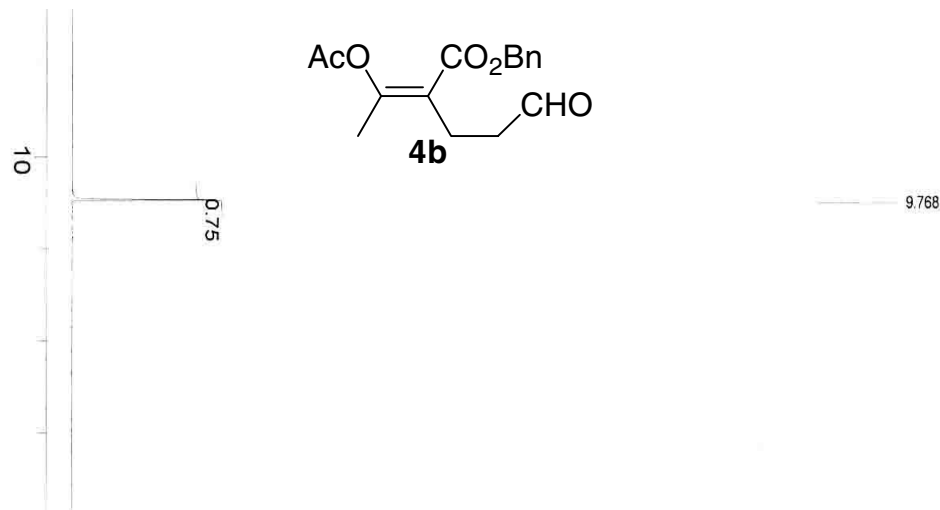

$\infty$
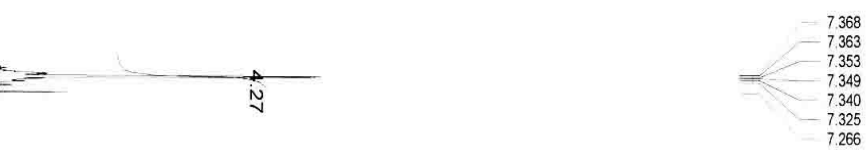

$a$

$A$

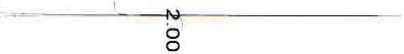

5.146

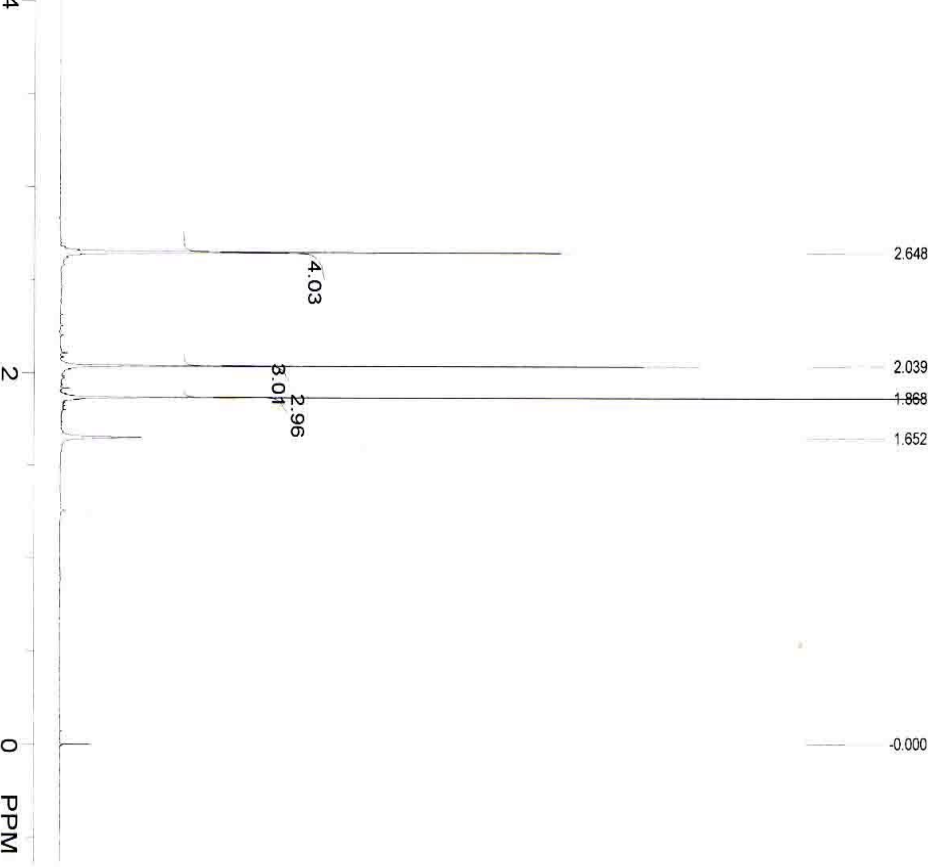




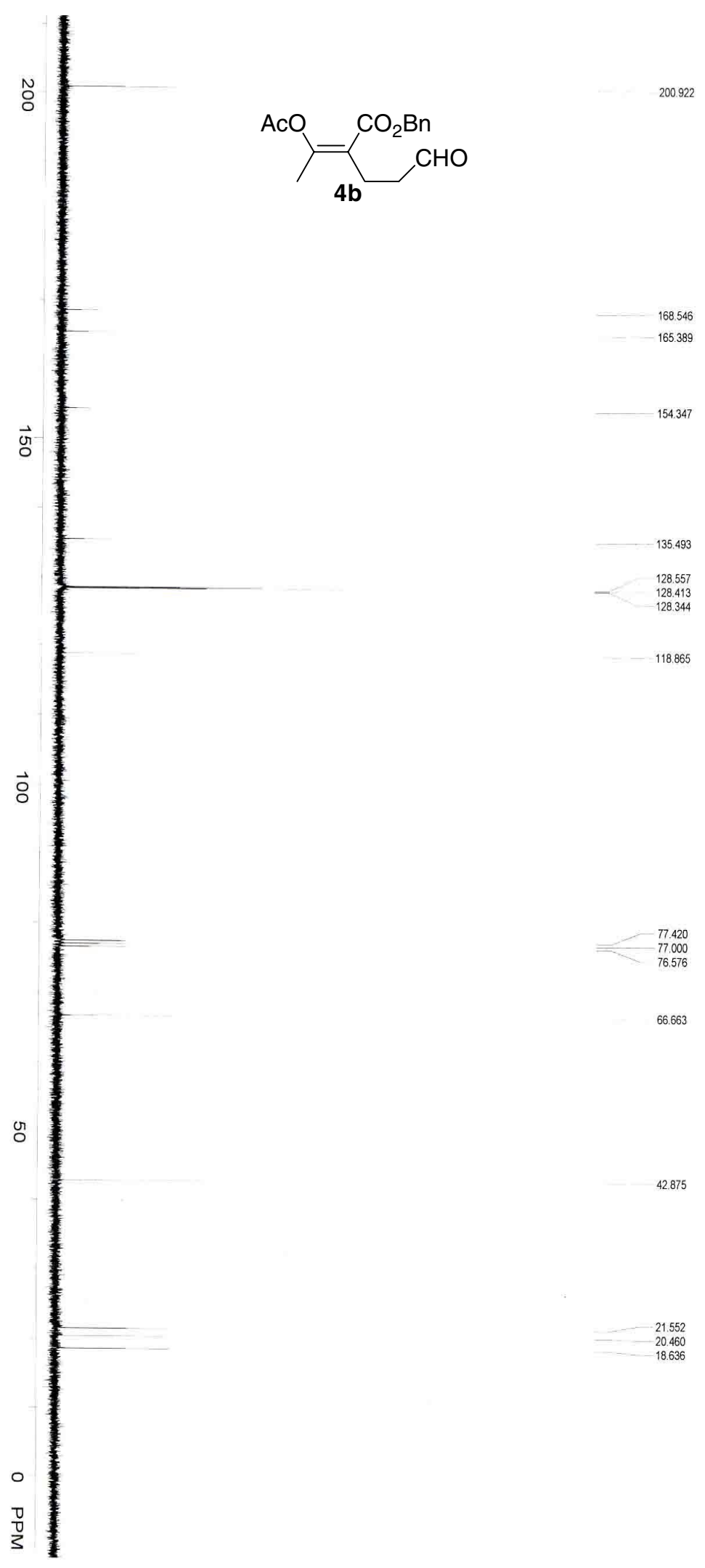




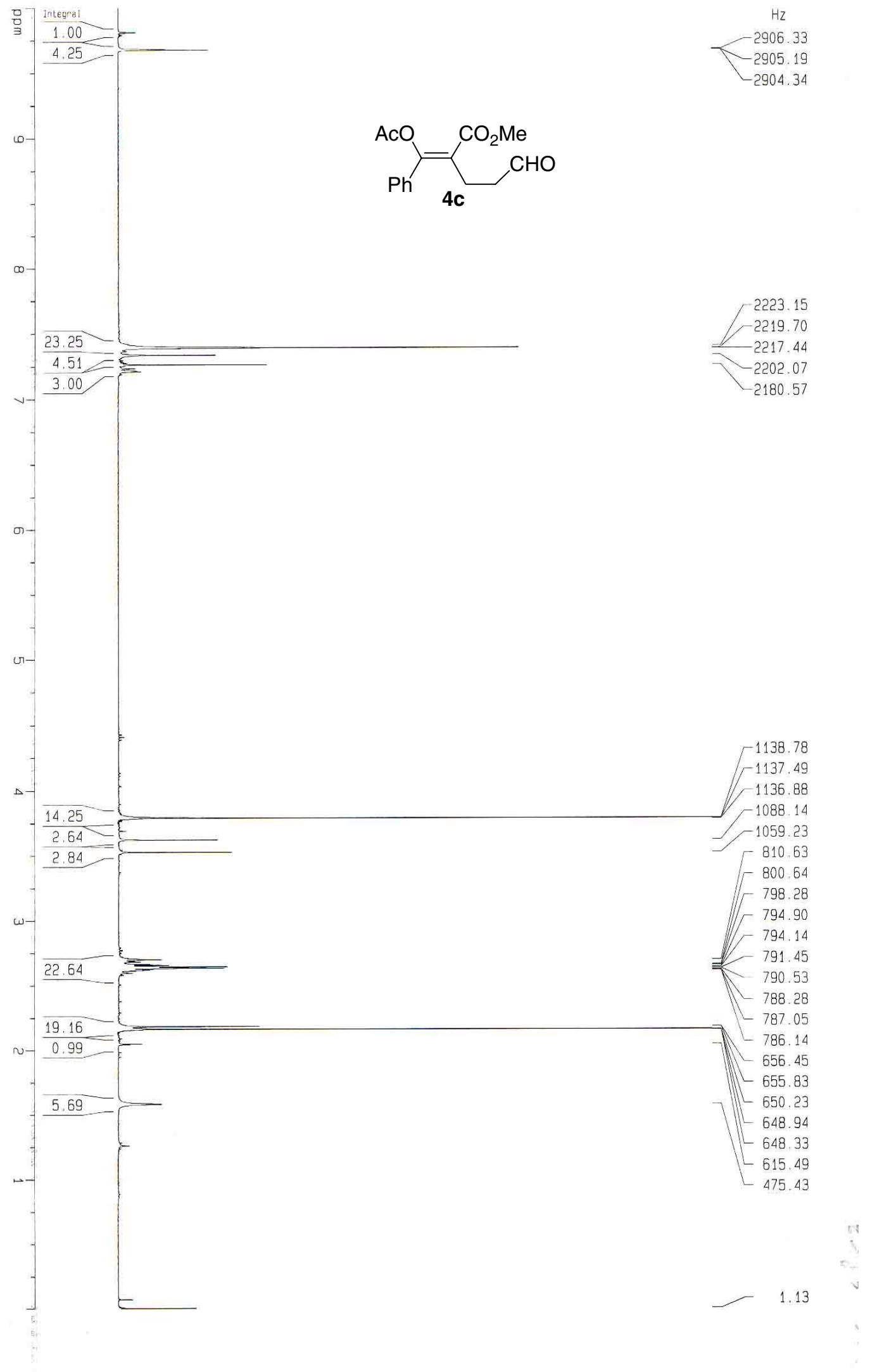




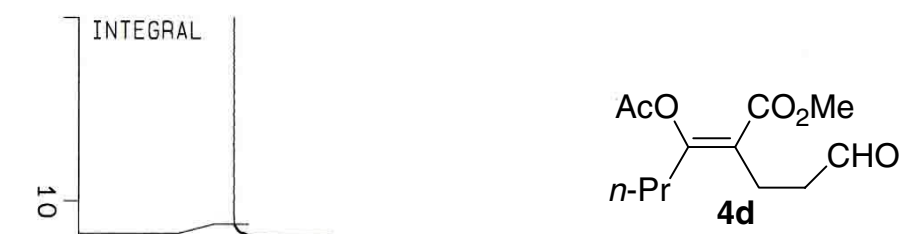

HERTZ

2940.32

2180.35

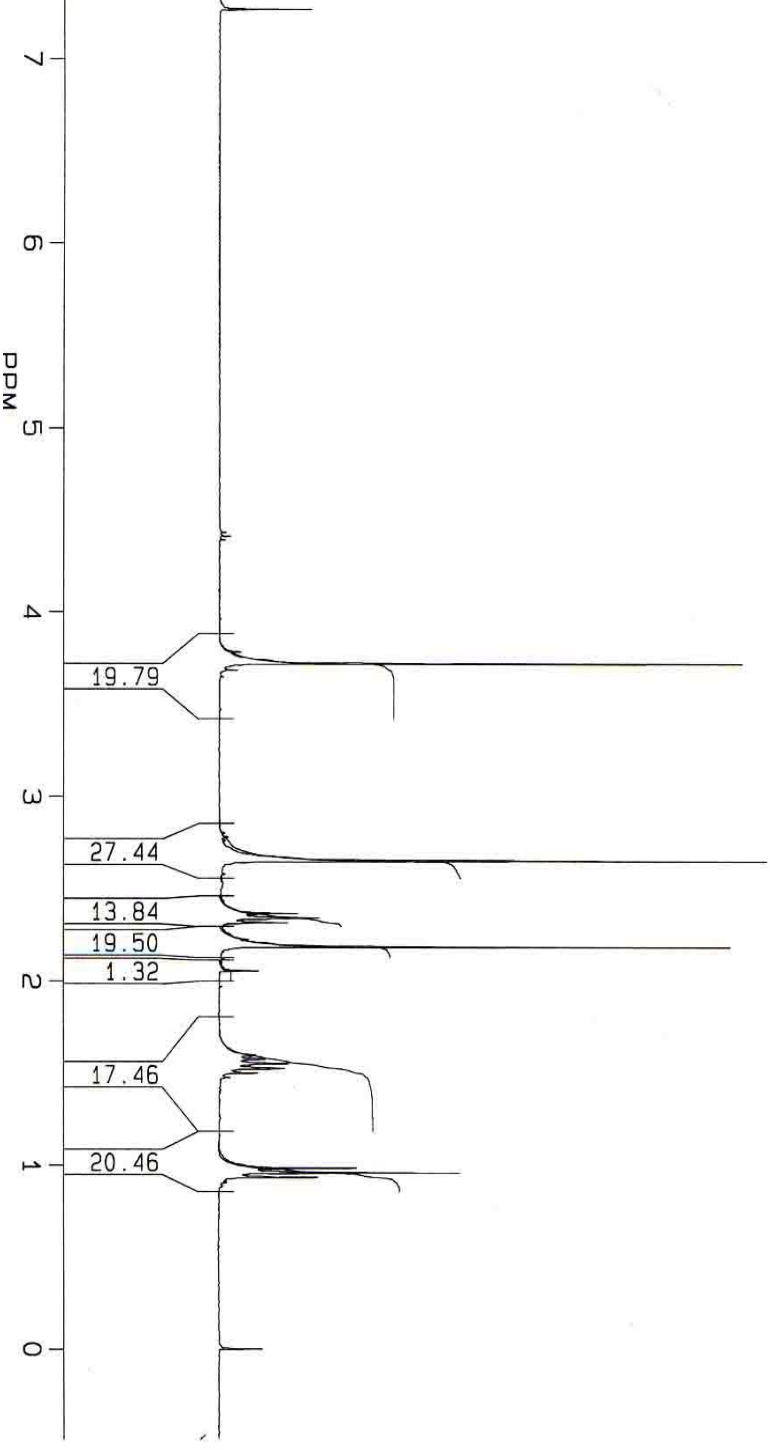

1119.82
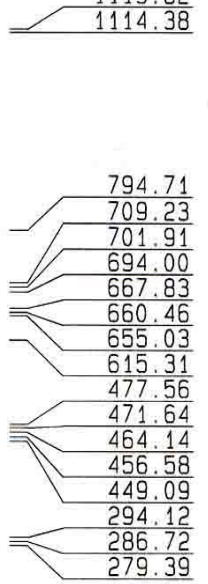

$-.61$ 


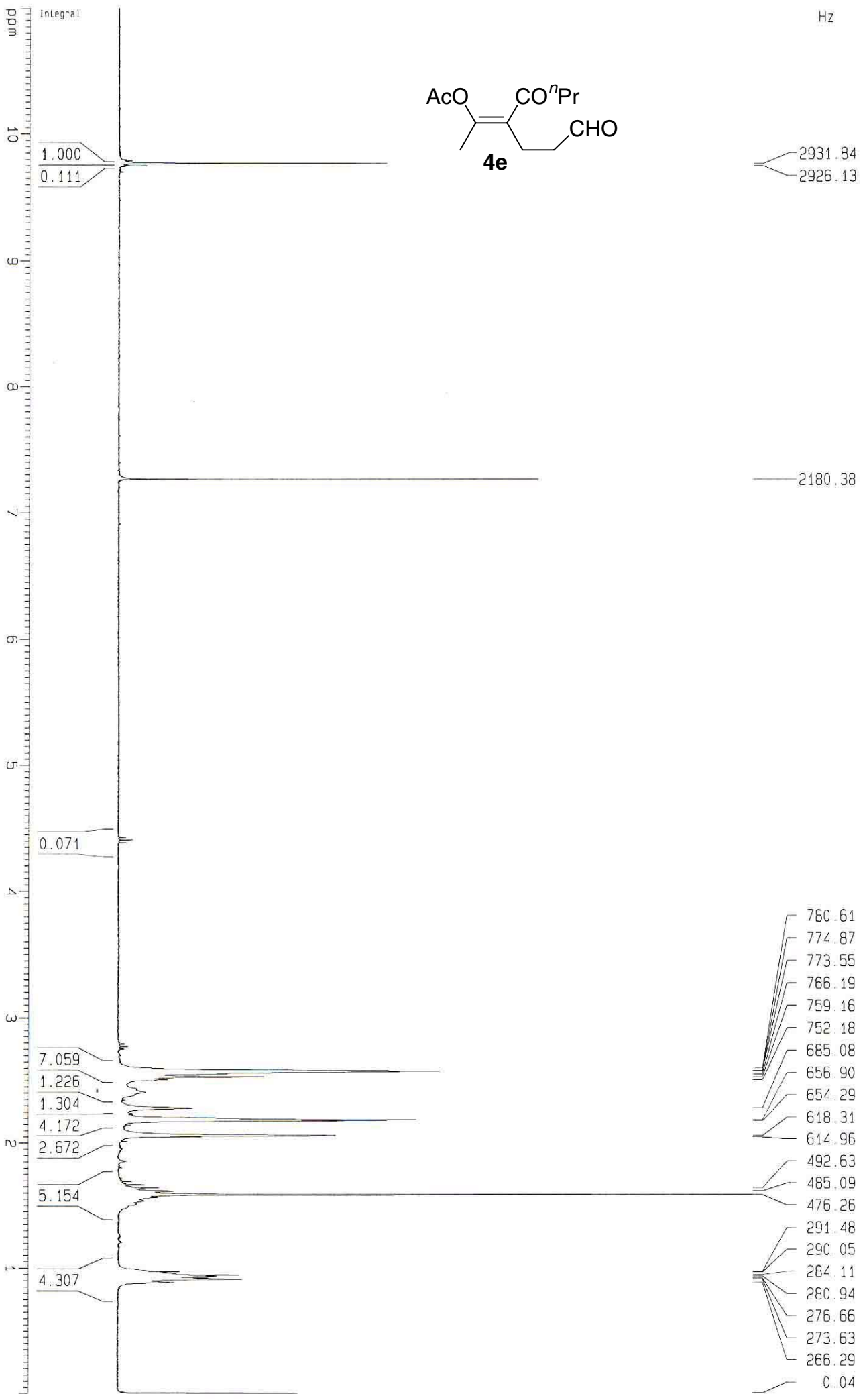



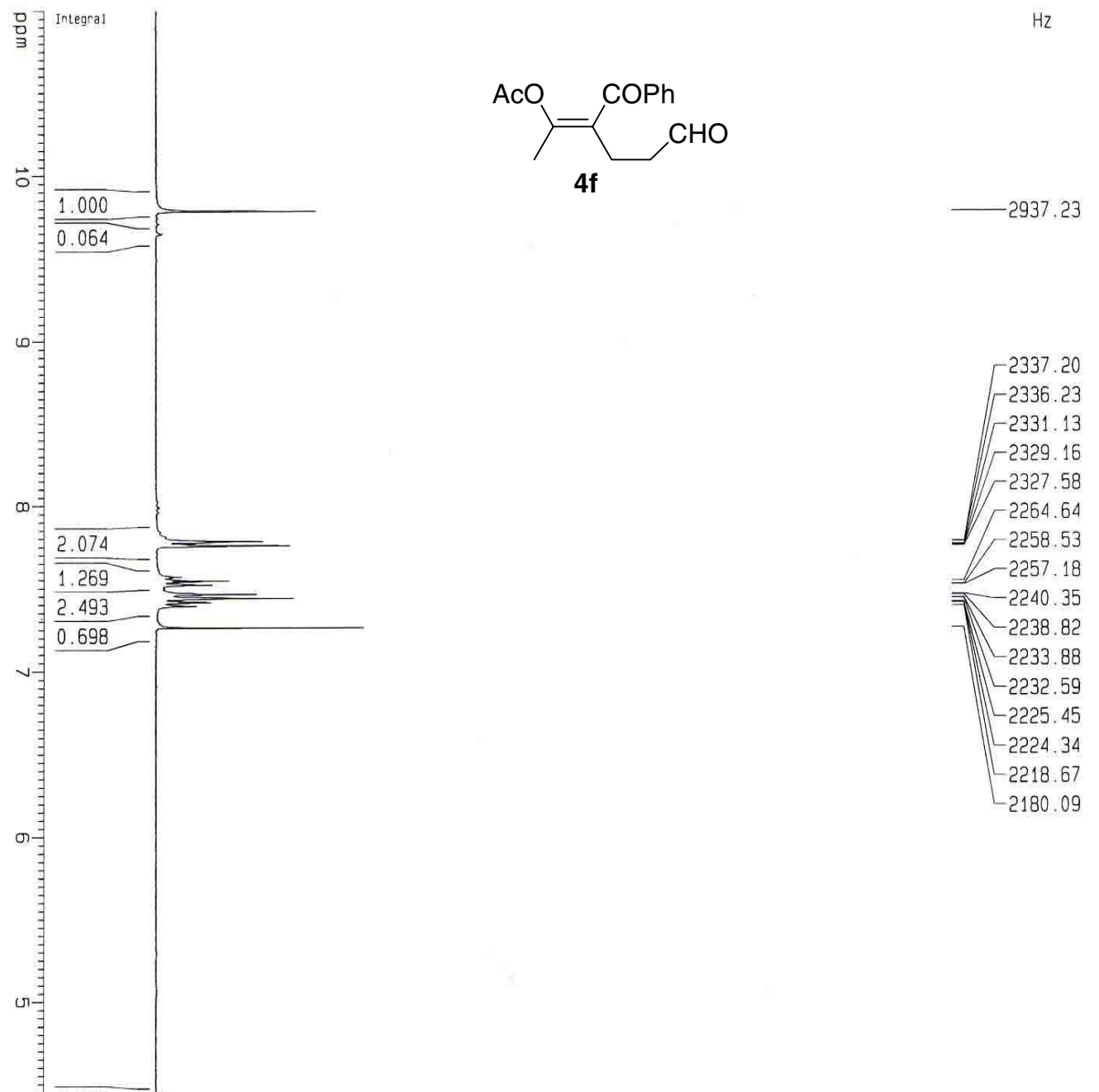

2180.09

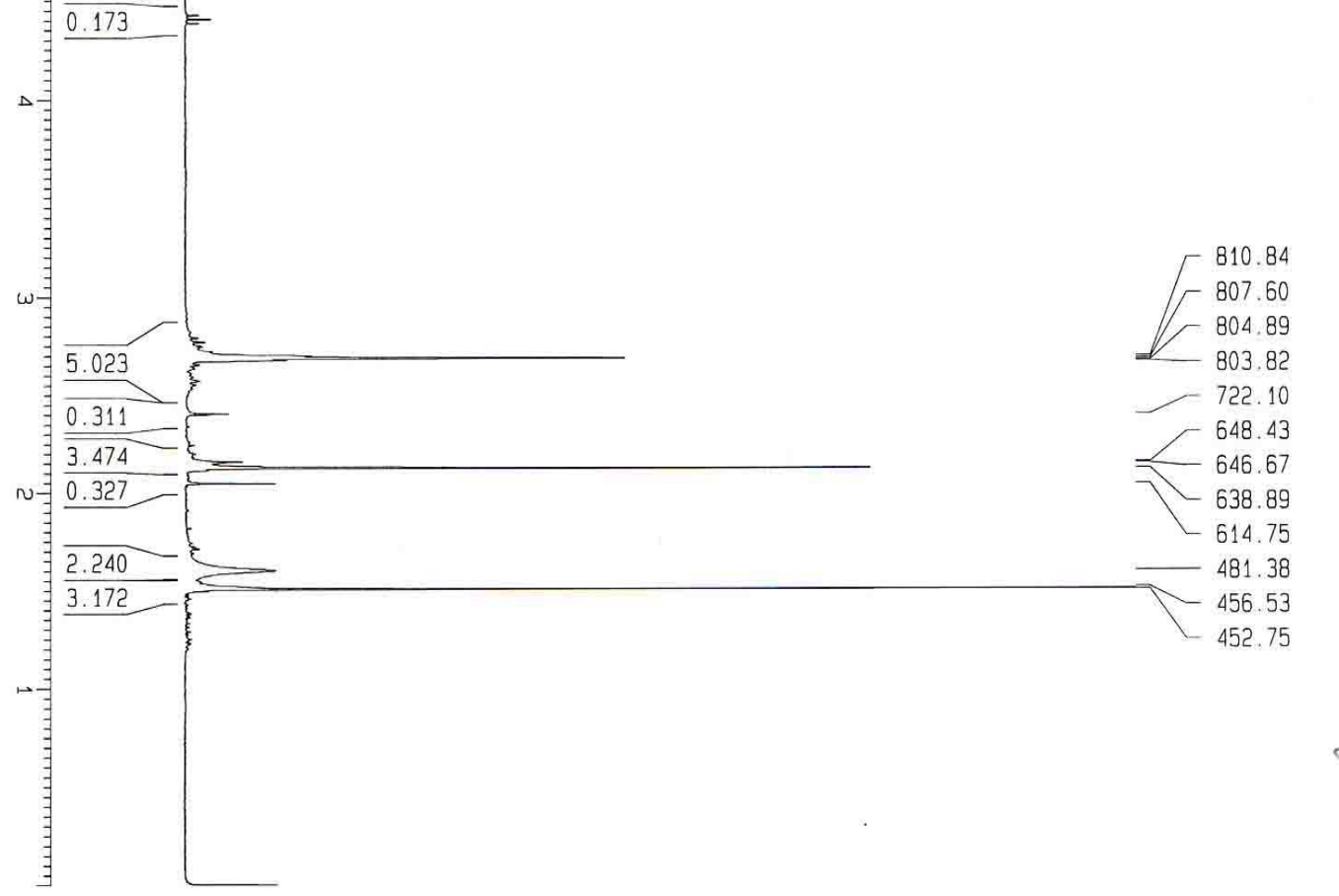




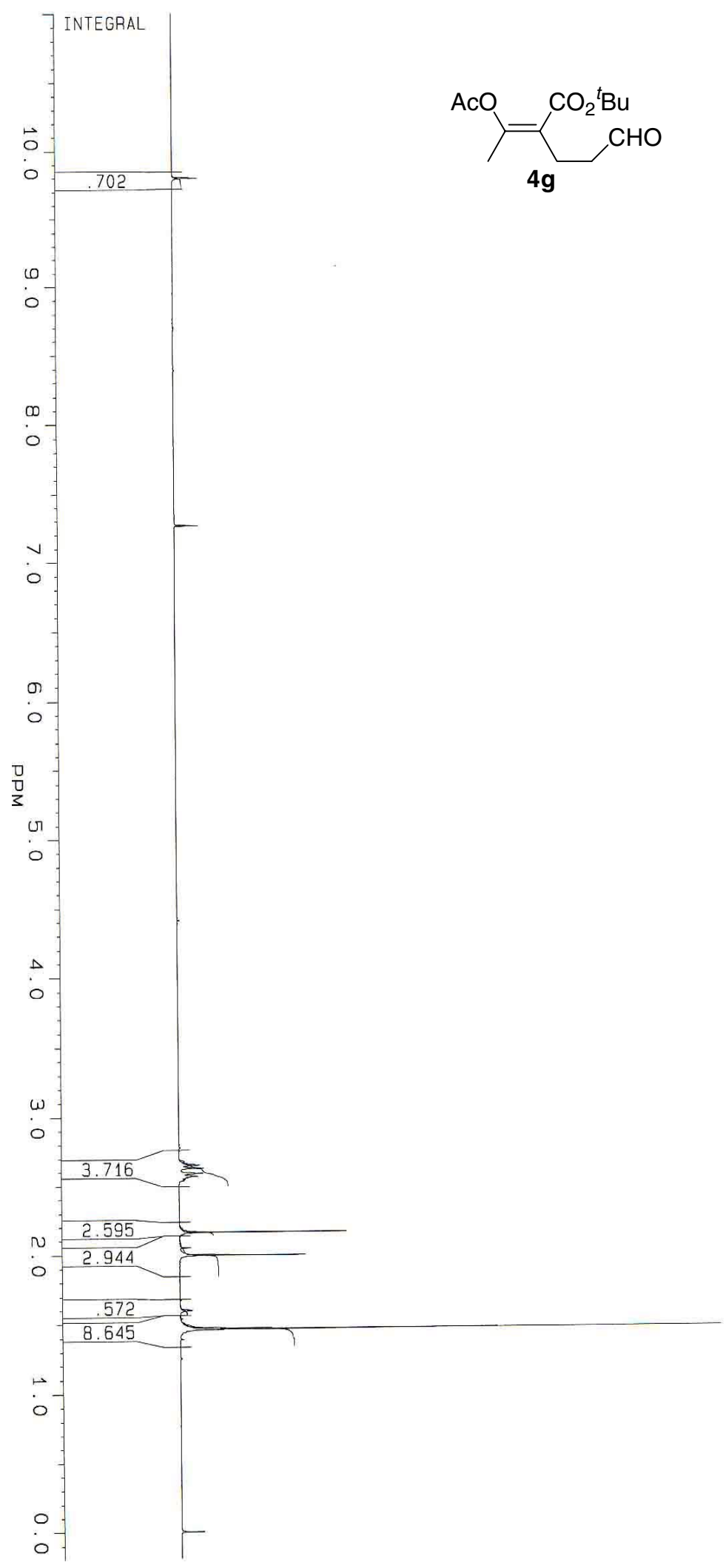

HERTZ

2940.13

2179.78

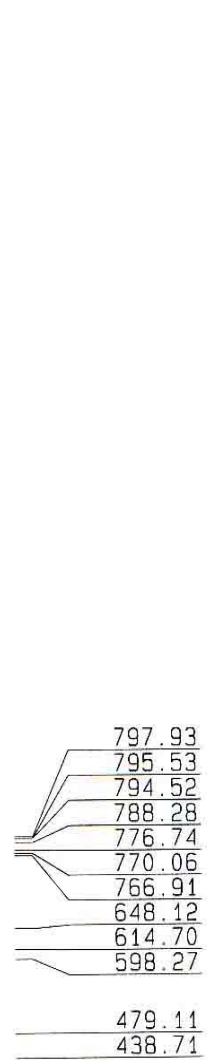

$-1.13$ 

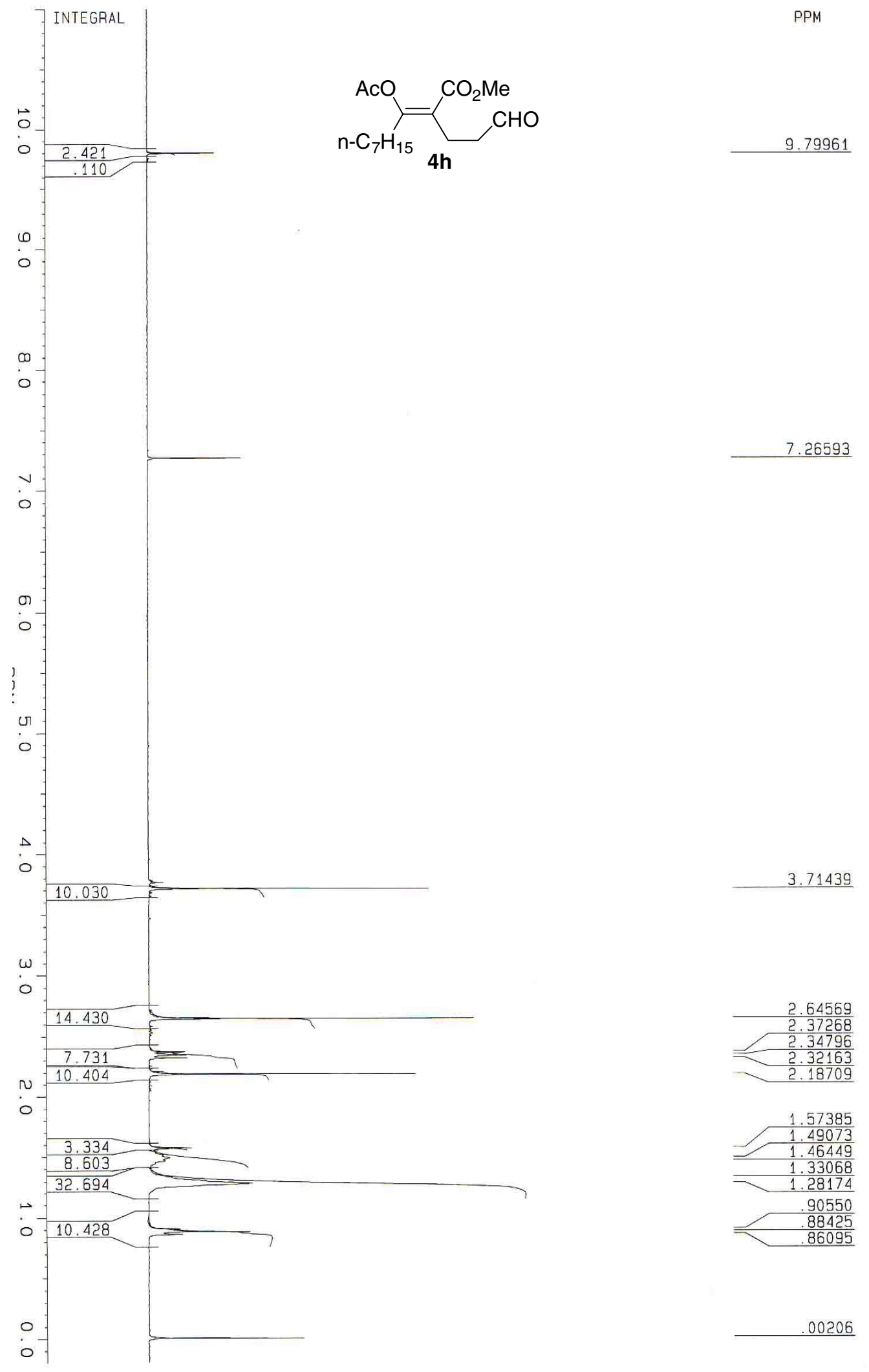


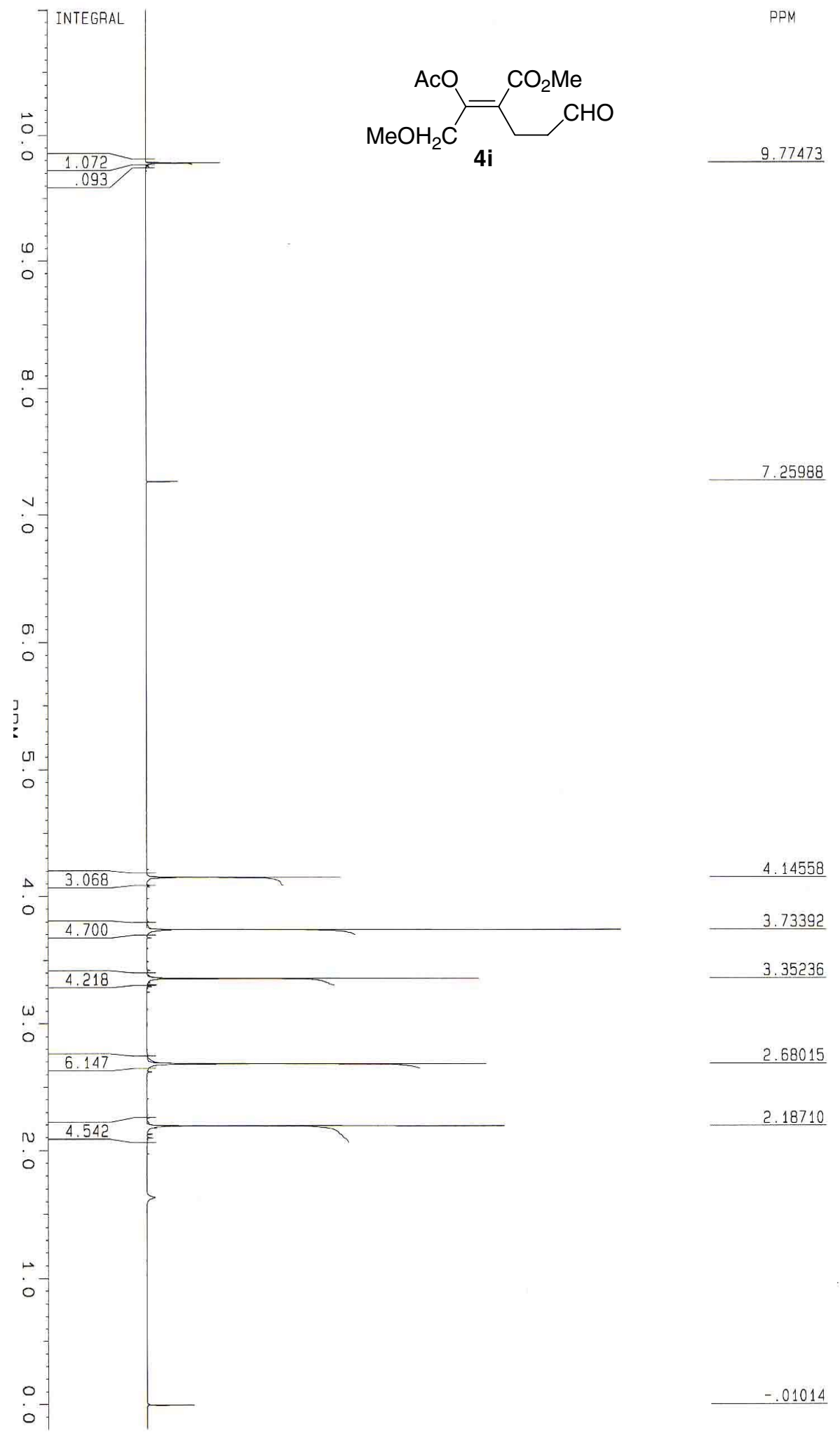




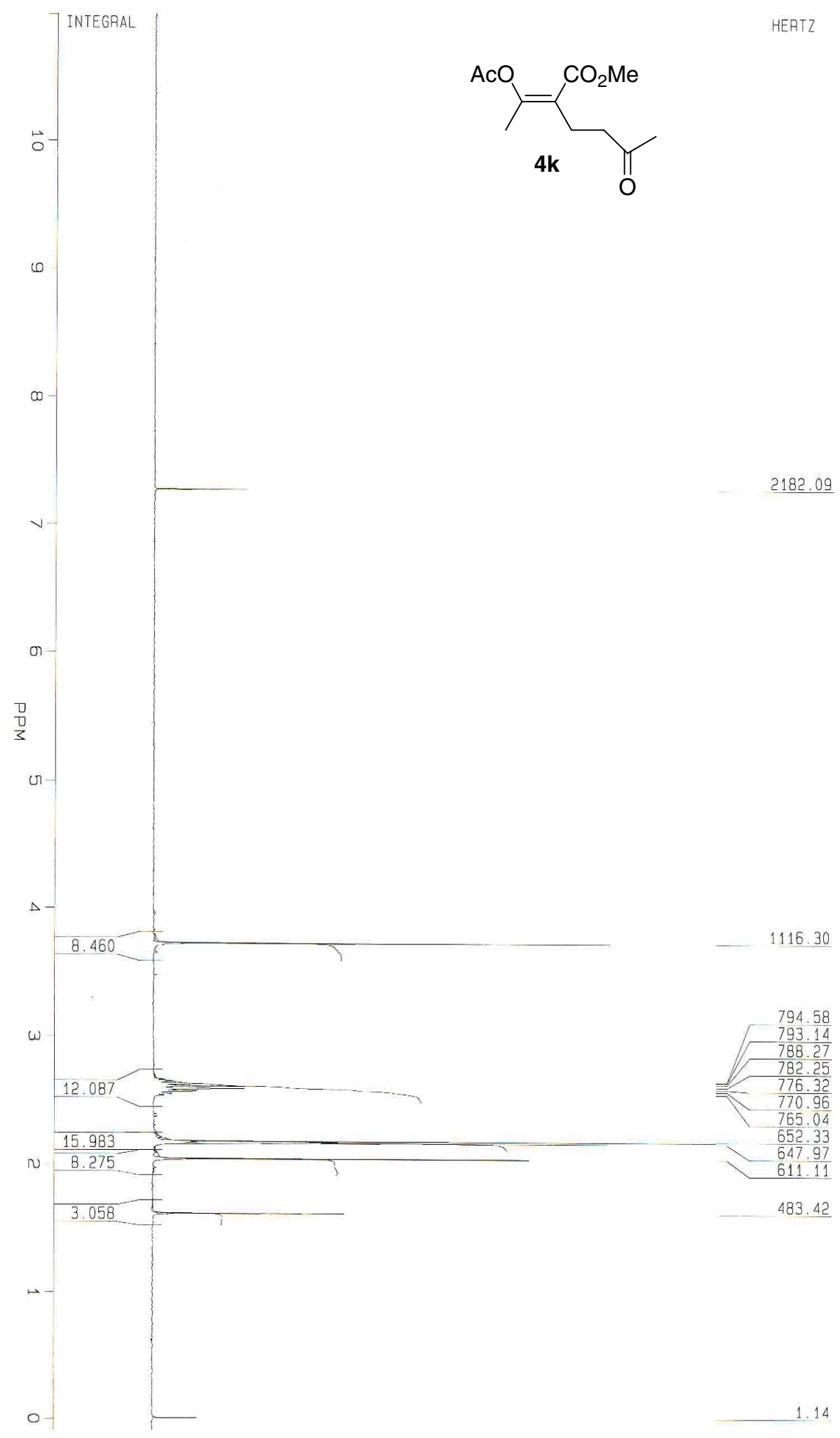




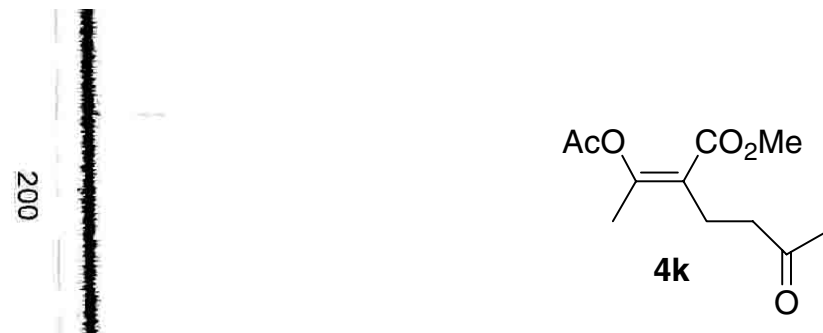

207529

153.829

방

119.119

59693

gr

$\overrightarrow{8}$

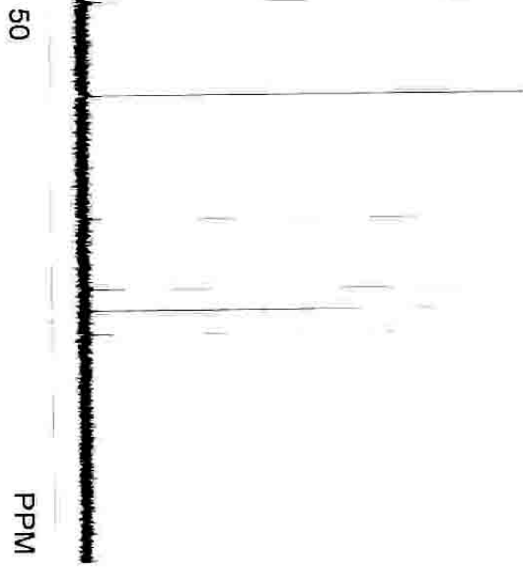

42342

29.921

22892

18422 